\title{
Research on Multi Frequency Vibration Test Method of Transport Package Based on Displacement Synthesis
}

\author{
Li-Ming Shen, Zhen-Wei Hu, Chao Wang \\ School of Mechatronics Engineering and Automation, Shanghai University \\ Shanghai 200072, China \\ E-mail: huzhenwei26@126.com
}

\begin{abstract}
By analyzing the existing vibration test method, include, sinusoidal frequency test method, sinusoidal frequency conversion test method and random vibration test method, we propose multi frequency vibration test method based on displacement synthesis. The new multi frequency vibration test table contain multiple mechanical rotating mechanisms that have different eccentricities, it cover wide frequency, output accurate and reliable frequency. With the new multi frequency vibration test table, the frequency that conform actual vibration can be simulated and output. It is a optimization for the original frequency test method.
\end{abstract}

Keywords-multi frequency vibration test method; vibration test table; vibration simulation

\section{INTRODUCTION}

From the production plant to reach the destination, the transportation process of production include several logistics links such as Loading and unloading, handling, transportation and storage. The vibration caused by transport, loading and unloading caused by the impact, they are easy to cause damage to the production [1-2]. Therefore, the vibration test is a main means of scientific reduction of commodity packaging, not only be propitious to reduce packaging costs, but also save resources, energy and environmental protection, it is widely used in the fields of transport packaging products, the vibration testing of transport packages of products [3-7] is an effective means of reliability evaluation and the effect of packaging products.

Therefore, packaging technology academia have paid long-term efforts around the standard test corresponding to existing transport packages of fixed frequency vibration and sine frequency sinusoidal vibration and random vibration of the three test methods [8-10], each revision strive synchronously with the test standards of ISO, ISTA, ASTM in other developed countries and region. Nevertheless, transport package vibration test standard in practical application has not been promoted effectively, most of the products packaging design base on the empirical data, or employ excessive packaging directly to replace the vibration test. Some famous enterprises that have vibration test equipment or even continue to use the actual vehicle and other methods to test the anti-vibration protection ability of the packaging of productions, this way not only takes a long test period, high cost and huge road resources, but also output discrete test results with low reliability.

Therefore, trough depth analysis of the existing transport package vibration test method, propose breakthrough and innovation in theory and method will become the effective way to reverse the passive situation, strive to transform the traditional vibration test into the more accurate and reliable quantitative analysis.

The existing three transport packing vibration test standards are respectively based on the technical basis of the mechanical rotating type vibration table or the electromagnetic and electro hydraulic shaking table [11-14]. The former mechanical rotating sinusoidal vibration table, its vibration output is clear and reliable, this meet the basic requirements of the popularization and application. However, the reference value of the experimental results is very limited because its test result is very different from the actual distribution of the frequency range of vibration. The latter electromagnetic and electro hydraulic shaking table, which is opposite to the former, its vibration spectrum can cover the actual vibration environment, but the vibration output is not clear, the result of the test has a greater uncertainty. Because there are different degree of defects in the existing transport packaging vibration test; the difference between the actual flow spectrum is large; the discreteness of the test result is large, it don't help significantly for the optimization of transport packaging design. Therefore, how to make a breakthrough in the vibration test technology, explore a vibration test table that can output definite vibration parameters, cover vibration frequency range of actual circulation process has become key to the effective promotion of vibration test of transport package in the future.

Analysis of Multi Frequency Vibration Test Method

In this paper, the author proposes a patent for mechanical vibration test table based on displacement synthesis. It mainly solute the fatal weakness of a narrow frequency range of the traditional mechanical rotary shaking table.

\section{MULTI FREQUENCY VIBRATION TEST METHOD}

A. Theory Analysis of Displacement Synthesis Test Method

Structure theory of traditional mechanical rotary shaking table is shown in Figure 1. 


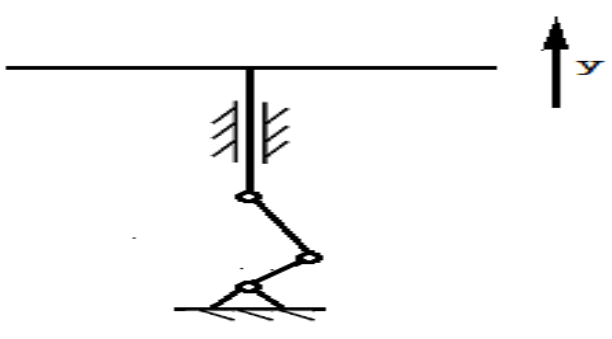

Figure 1. Structure theory of traditional mechanical rotary shaking table.

Vibration displacement y:

$$
\mathrm{y}=y_{0} \cos (\omega t+\varphi)
$$

Corresponding vibration acceleration:

$$
\mathrm{a}=\mathrm{y}_{0} \omega^{2}(\omega \mathrm{t}+\varphi)
$$

Obviously, the acceleration peak value $\mathrm{A}$ is twice of the vibration frequency $\mathrm{f}$, that is:

$$
\mathrm{A}(\omega)=\mathrm{kf}^{2}
$$

Among them, $\mathrm{k}$ is a constant, which is determined by the displacement amplitude y0, that is, the eccentricity of the vibration mechanism.

According to the analysis of the vibration environment, the main vibration amplitude is in the range of $0 \mathrm{~Hz}$ to $100 \mathrm{~Hz}$. Among them, the amplitude of low frequency vibration displacement is large, the corresponding peak value is about $20 \mathrm{~mm}$. The displacement amplitude decreases with the increasing frequency, the displacement peak value which close to $100 \mathrm{~Hz}$ is far less than $1 \mathrm{~mm}$, the vibration is higher than $100 \mathrm{~Hz}$ is absorbed by the buffer packaging structure and suspension system of packaging.

Comprehensive analysis of the above findings, any single eccentric distance mechanical rotation vibration can't meet the two requirements of displacement amplitude and frequency range simultaneously. So, can you use a combination of mechanical rotating mechanism with different eccentric distance to meet the requirement of displacement amplitude and frequency range synchronously?

Acceleration amplitude $\mathrm{A}$ and frequency $\mathrm{f}$ curves of different eccentricity mechanism is shown in figure 2. Each (left to right) displacement amplitude graph is corresponding to the y0, y1...yi. Yi is decided by eccentricity, the relation is $\mathrm{y} 0>\mathrm{y} 1>\ldots>\mathrm{yi}$.

By assigning different $\mathrm{Yi}$ values, each curve cover different frequency ranges. In the figure, the y0 curve covers f0 to fi, y1 curve covers f1 to fi...Obviously, this method can theoretically make mechanical rotary vibration table wide in the frequency range, but to practical application, the mechanism which can make the linear synthesis of the vibration displacement on behalf of each curves is key, it can output vibration frequency of different eccentricity in the same plane.

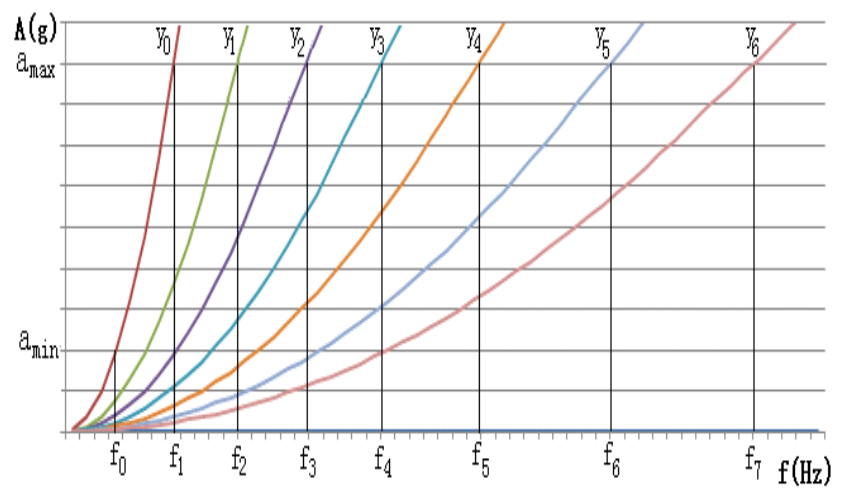

Figure 2. Acceleration amplitude A and frequency f curves.

\section{B. Theory of Mechanical and Institutional}

According to studies, compound a plurality of mechanical rotating vibration displacement to a synthetic mechanism with multiple degrees of freedom is very complex for both institutional theory analysis and manufacturing process because vibration displacement is space vector. So, packet simplification is a common method to analysis and design complex mechanism. The four bars differential mechanical lever mechanism can compound displacement of vertical component of two rotational vibrations easily, that is shown in figure 3 .

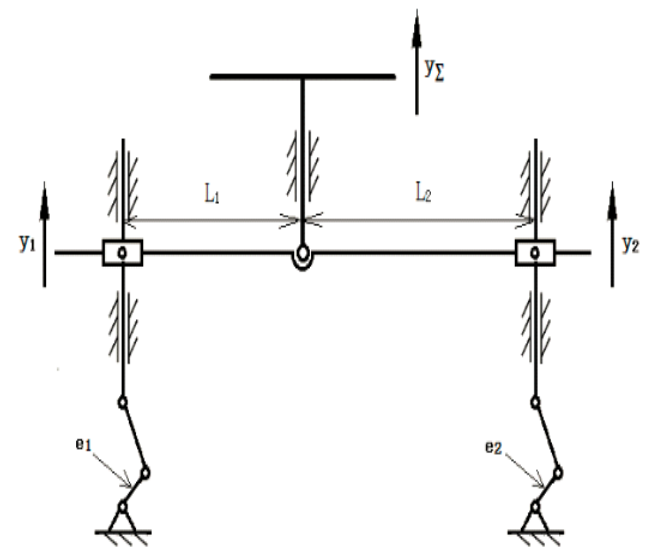

Figure 3. Composite graph of displacement of vertical component of two rotational vibrations.

The synthesis of vertical vibration $\mathrm{y}_{\Sigma}$ :

$$
\begin{gathered}
\mathrm{y}_{\Sigma}=\mathrm{k}_{1} \mathrm{y}_{1}+\mathrm{k}_{2} \\
k_{1}=\frac{L_{1}}{L_{1}+L_{2}}, \quad k_{2}=\frac{L_{2}}{L_{1}+L_{2}} .
\end{gathered}
$$

When the vibration synthesis lever arm is much larger than eccentricity ratio, namely L >>e, crank and connecting rod mechanism can be used to replace, that is shown in figure 4, the movable hinge with vibrational excitation on both ends of the lever can be simplified as a fixed hinge. 


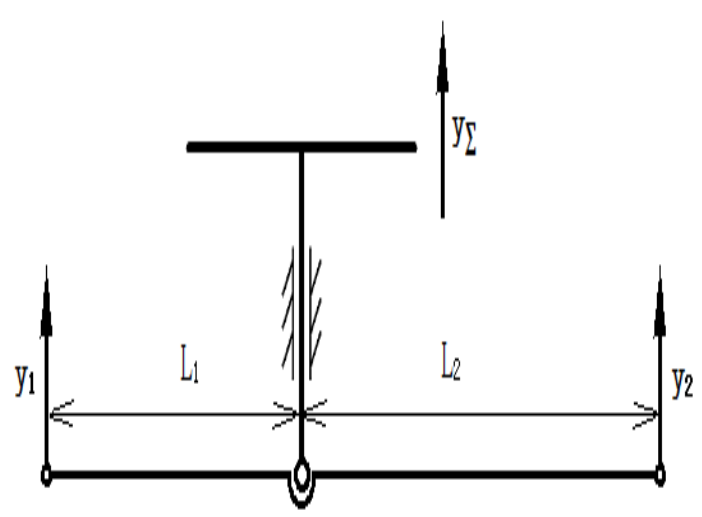

Figure 4. The fixed hinge model diagram.

Use the other group of similar vibration synthesis lever mechanism to replace any one end of original lever of vibration exciting connecting rod, then the end of original lever of excitation vibration is decomposed into two sub vibrations. The vibration test table is formed displacement vectors synthesis by three mechanical eccentric rotation mechanisms. That is shown in figure 5 .

The composite displacement $y_{\Sigma}$ :

$$
\begin{gathered}
\mathrm{y}_{\Sigma^{3}}=\mathrm{k}_{1} \mathrm{y}_{1}+\mathrm{k}_{2}\left(\mathrm{k}_{21} \mathrm{y}_{1}+\mathrm{k}_{22} \mathrm{y}_{3}\right) \\
k_{21}=\frac{L_{3}}{L_{3}+L_{4}}, k_{22}=\frac{L_{4}}{L_{3}+L_{4}}
\end{gathered}
$$

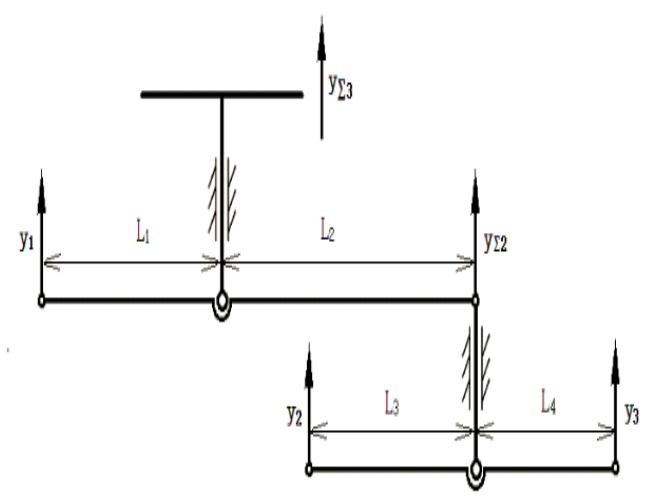

Figure 5. The synthesis diagram of three rotation mechanism structure.

In this way, practical mechanism of vibration displacement synthesis of $\mathrm{N}$ mechanical rotation vector can be constructed in theory; vibration of different frequencies generated by n eccentric mechanism is synthesized on multi frequency vibration table. The structure is shown in figure 6 .

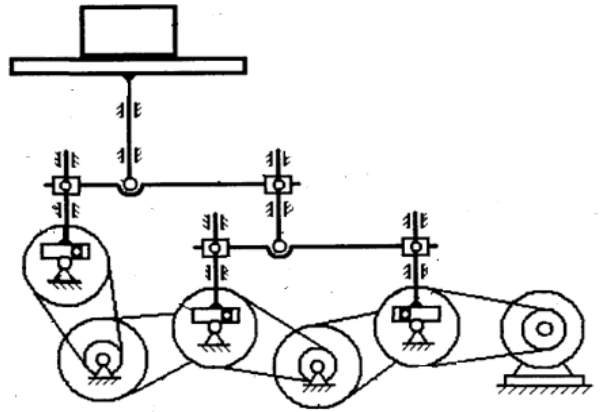

Figure 6. The structure of Multi Frequency Vibration Test Table.

\section{CONCLUSION}

In this study, through in-depth analysis of the existing transport packaging vibration test standard, the bottleneck problem of its application is summarized. The principle and characteristics of the multi frequency vibration test table are expounded in the paper. The creativity, practicality and advance of multi frequency vibration test technology are demonstrated, and the feasibility of the technique is demonstrated by comparison between theoretical value and experimental value. The practical significance of the multi frequency vibration test technology in the field of transport packaging is verified by the results of the prototype, and lays the foundation for the research of the standard of the future transport packaging parts multi frequency vibration test method.

\section{REFERENCES}

[1] Xu Wencai, Xiang Ming. Effects of continuous impact loading on packaging products [J]. vibration and shock, 2001,20 (3): 26-28.

[2] Meng Xianwen, Ji Hongwei, Wang Huaiwen, et..PC host transportpackaging parts for the evaluation of vibration and shock, [J] vibration and shock, 2007, 26 (8).

[3] Tong Yan Tee,Hun Shen Ng. Impact life prediction modeling of TFBGA packages under board level drop test[J]. Microelectronics Reliability,2004.44:1131-1142

[4] Desmond Y R, Chong F X Che Drop impact reliability testing for leadfree and lead-based soldered IC packages [J]. Microelectronics Reliability, 2006, 46: 1160-1171.

[5] Jeong-WookYi, Gyung-JinPark Development of a design for EPS cushioning package of a monitor using axiomatic design[J].Advances in Engineering Software,2005,36:273-284.

[6] Low K H,Wang Yuqi,Hoon K H. A virtual boundary model for a quick drop-impact analysis of electronic components in TV model [J]. Advances in Engineering Software, 2004, 35:537-551.

[7] Wang P.L. Studies on diffusion coefficients of migrants in plastic packaging by molecular dynamics[D]. Jinan University, 2010.

[8] The part of the national standard [S].GB/T4857.7-2005. packaging and transport packaging part of the basic test of the seventh part: the sine fixed frequency vibration test method.2005

[9] The tenth part of the national standard [S].GB/T4857.10-2005. packaging and transport packaging part: sinusoidal frequency conversion vibration test method.2005.

[10] Random vibration test method of national standard [S].GB/T4857.232003. package and transport package in People's Republic of China.2003.

[11] Wu Wu. Practical vibration test technology [M]. Beijing: Weapon Industry Press, 1993. 
[12] Hu Zhiqiang, law Qing Yan, Hong Baolin, et al. Application of random vibration test technology [M]. Beijing: China Metrology Publishing House: 1996
[13] Zhang Qiaoshou. The vibration test system of the status quo and development of [J]. aerospace technology and civilian products, 2000.

[14] Benzoni G. Challenges of new generation seismic testing facilities [J]. Experimental techniques, 2001, March /April: 20-23. 